\title{
Outbreak of candidemia caused by fluconazole resistant Candida parapsilosis strains in an intensive care unit
}

\author{
Henrique Marconi Sampaio Pinhati ${ }^{1 *}$, Luiz Augusto Casulari ${ }^{1}$, Ana Carolina Remondi Souza², \\ Ricardo Andreotti Siqueira ${ }^{2}$, Camila Maria Gomes Damasceno ${ }^{1}$ and Arnaldo Lopes Colombo ${ }^{2}$
}

\begin{abstract}
Background: Candidemia is an increasing problem in tertiary care hospitals worldwide. Here, we report the first outbreak of candidemia caused by fluconazole-resistant C. parapsilosis (FRCP) strains in Brazil.

Methods: This was a cross-sectional study of clinical and microbiological data of all candidemic episodes diagnosed from July 2011 to February 2012 in a 200-bed tertiary care hospital. Initial yeast identification and susceptibility testing were performed using the VITEK 2 - System. Isolates of Candida spp. resistant to fluconazole were sent to a reference laboratory (LEMI-UNIFESP) for further molecular identification and confirmation of resistance by CLSI microdilution test. A multivariate analysis was conducted to identify factors associated with FRCP infection.

Results: We identified a total of 40 critically ill patients with candidemia (15 women) with a median age of 70 years. The incidence of candidemia was 6 cases/1,000 patients admissions, including 28 cases (70 \%) of infection with C. parapsilosis, 21 of which (75\%) were resistant to fluconazole. In only $19 \%$ of FRCP candidemia cases had fluconazole been used previously. The results of our study indicated that diabetes is a risk factor for FRCP candidemia ( $p=0.002)$. Overall, mortality from candidemia was $45 \%$, and mortality from episodes of FRCP infections was $42.9 \%$.
\end{abstract}

Conclusions: The clustering of incident cases in the ICU and molecular typing of strains suggest horizontal transmission of FRCP. Accurate vigilant monitoring for new nosocomial strains of FRCP is required.

Keywords: Candida sp, Candidemia, Fluconazole resistance, Antifungal treatment

\section{Background}

Hematogenous candidiasis is an alarming problem in tertiary care hospitals worldwide, especially in patients admitted in intensive care units (ICU) [1, 2]. Incidence rates of candidemia vary considerably among geographic areas and medical centers. For example, in Europe and the USA, different studies have reported incidence rates of $0.2-1.87$ and $0-2.4$ per 1,000 admissions, respectively $[3,4]$. In Brazil and Latin America, nosocomial candidemia is an important problem in adults and children, and the reported incidence rates are $0.33-6$ cases per 1,000 hospital admissions [5-7]. The large variation in the incidence rates of candidemia is probably due to a

\footnotetext{
* Correspondence: hpmarconi@gmail.com

${ }^{1} E d$. América Office Tower, SCN Q 1 BL F, - sala-1016, Asa Norte, Brasília, DF 70711-905, Brazil

Full list of author information is available at the end of the article
}

combination of several factors, including differences in resources available for medical care and training programs, difficulties in the implementation of infection control programs in hospitals in developing countries, limited number of health care workers to assist patients in critical care units, and less-aggressive practices of empirical antifungal therapy and prophylaxis for high-risk patients [6]. Hospital-acquired candidemia is associated with a large increase in hospitalization duration, attributable mortality (up to $40 \%$ ) and costs $[8,9]$.

Although Candida albicans is still considered the main causative species of candidemia worldwide, there is a concern over the rise in invasive infections caused by non-albicans species. In this regard, Candida glabrata has frequently been reported in Northern Europe and US medical centers $[1,10]$, whereas Candida parapsilosis and Candida tropicalis are the most common non- 
albicans species associated with fungemia in Latin America and Asia [11, 12]. Of note, a recent multicenter study in the USA found that $C$. parapsilosis was the second most commonly encountered non-Candida albicans species [13].

Fluconazole is accepted as an alternative treatment for patients with candidemia due to its efficacy and safety demonstrated by several clinical trials $[14,15]$. For candidemia caused by Candida parapsilosis complex, in particular, fluconazole has been suggested as the best alternative for primary therapy [16]. There are some controversies in the literature regarding the effectiveness of echinocandins against infections of $C$ parapsilosis, as high MICs are usually observed when this species is tested against drugs of this class [17].

During July-2011 and February-2012, we observed a dramatic increase in the incidence of candidemia reported in ICU patients from a single institution compared to the previous period between January-2006 and June-2011, where $70 \%$ of all episodes enrolled C. parapsilosis strains (Fig. 1). We were able to type 09 out 28 C. parapsilosis strains enrolled in this outbreak and they were considered genetically related [18]. Further antifungal susceptibility tests confirmed that most isolates were resistant to fluconazole. The present publication describes clinical and epidemiological data related to this outbreak of fluconazole resistant C. parapsilosis candidemia complementing information that was not explored in our previous report on molecular typing and resistance mechanisms related to the mentioned strains [18].

\section{Methods}

We conducted a cross sectional study of clinical and laboratory data of all adult patients (age $\geq 18$ years) diagnosed with candidemia during their hospitalization in intensive care unit between July, 2011 and February, 2012 (eight months period) to investigate a candidemia outbreak. Our local ethical committee approved the protocol (CEP: 73/2011).

\section{Laboratory procedures}

Yeast identification and antifungal susceptibility testing were initially performed by the local microbiology laboratory using the VITEK 2 - system (bioMérieux, Marcy-I'Étoile, France). All C. parapsilosis isolates stored at the routine laboratory were sent to a reference laboratory (LEMI - Laboratório Especial de Micologia, Universidade Federal de São Paulo, São Paulo-SP, Brazil) for further molecular identification and confirmation of antifungal susceptibility testing by the Clinical and Laboratory Standards Institute (CLSI) reference method.

(i). Molecular identification of C. parapsilosis (sensu lato) isolates by real time TaqMan ${ }^{\circ} \mathrm{PCR}$ assays. DNA was extracted from the isolates using mechanical disruption with glass beads and phenol/ chloroform [19]. Real-time PCR was performed using species-specific TaqMan ${ }^{\circ}$ probes, as previously described by our group [20].

(ii). In vitro susceptibility testing. Antifungal susceptibility testing was performed at the reference laboratory using the CLSI microdilution assay [21]. Fluconazole and anidulafungin were provided by Pfizer Pharmaceutical Group (New York, NY, USA) and amphotericin B was provided by Sigma Chemical Corporation (St. Louis, MO, USA). The CLSI-M27-S4 document was used as the gold standard interpretative guidelines for classifying the $C$. parapsilosis (sensu lato) isolates as susceptible, susceptible dose dependent (SDD)/intermediate, or resistant to azoles and echinocandins [22].

\section{Statistical analysis}

We performed univariate and multivariate analyses to identify risk factors associated with FRCP. For the

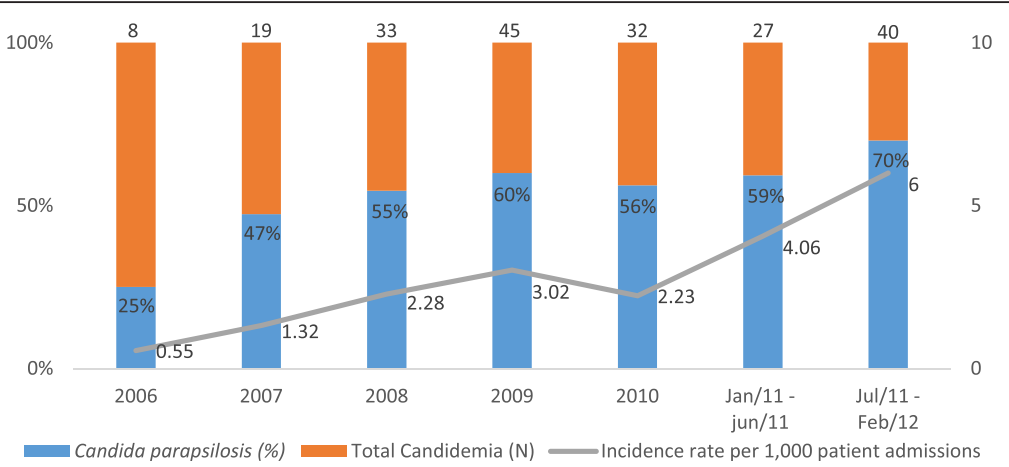

Fig. 1 Incidence rates of candidemia and prevalence of Candida parapsilosis in ICU patients admitted at a single institution between 2006 and 2012 
univariate analysis, categorical variables were compared using the chi-square or Fisher exact tests, and continuous variables were compared using the Student $t$ test or Mann-Whitney U test, as appropriate. All statistical tests were two-tailed, and the significance level was set at $p<0.05$.

After the initial univariate analysis, the variables with significant FRCP association were included in a multivariable analysis with adjustments for the covariates of age and gender. A Poisson regression with robust variance (linear-log) was employed for the multivariate analysis. The level of significance was set at $p<0.05$ [23].

\section{Results}

During the study period, 40 episodes of candidemia were reported in ICU patients. The median age of the patients was 70 years (range: $23-91$ years), and $37.5 \%$ of the patients were female. The overall incidence of candidemia during the eight month study period was 6 cases/1,000 patients-day admissions.

The VITEK 2 system identified 28 blood stream Candida isolates as C. parapsilosis (70 \%), 9 as C. albicans (22.5\%), 2 as C. glabrata (5\%) and 1 as C. tropicalis (2.5\%). Using the VITEK 2 system, fluconazole resistance or SDD was detected in 21 out 28 C. parapsilosis (75\%) isolates tested. All 9 C. albicans, 2 C. glabrata and 1 C. tropicalis strains were considered susceptible to fluconazole by the automated system. All 40 Candida spp strains were susceptible to amphotericin B and anidulafungin.

Fourteen out 28 clinical strains of C. parapsilosis isolates enrolled into the outbreak had been stored by the routine laboratory and were available to be sent to the reference laboratory (LEMI-UNIFESP) for further molecular identification and confirmation of antifungal susceptibility test results by the CLSI broth microdilution (BMD) reference method. Except for one clinical isolate identified as C. metapsilosis, all other strains were genetically identified as C. parapsilosis (sensu stricto) when tested using species specific TaqMan ${ }^{\circledR}$ probes. Despite some differences among MICs generated by the two methods, the categorical agreement between VITEK 2 and CLSI BMD was very good (72\%). A total of 11 out 14. $C$ parapsilosis strains categorized as SDD or resistant to fluconazole by the VITEK 2 system, were considered resistant or SDD when tested by the CLSI method. Of note, 9 of the 11 strains categorized as resistant to fluconazole by the BMD exhibited similar genetic profile by microsatellite assay and molecular mechanisms related to azole resistance as reported by our group elsewhere [18]. No strains of C. albicans, C. tropicalis or $C$. glabrata were available for testing by the CLSI methodology. No strains of $C$. parapsilosis were resistant to anidulafungin or amphotericin B as already documented by the VITEK 2 system.
Considering that only 14 of the 40 isolated strains were tested by the CLSI method, we used the information provided by the VITEK 2 system to check for risk factors associated with infection by fluconazole-SDD or -resistant Candida.

The epidemiological and clinical characteristics of the patients infected by FRCP strains and those infected by fluconazole-susceptible Candida (FSC) species are presented in Table 1. In general, most demographic and clinical data were similar between the two patient groups. The exposition to central venous catheter, hemodialysis, surgery, mechanical ventilation, antibiotics and corticosteroids, were similar in both groups. It is important to mention that only 4 of the 21 patients (19 \%) infected by FRCP had been previously exposed to fluconazole before developing candidemia compared to 3 of the 19 patients (15.8\%) infected by FSC strains $(p=1)$. After univariate analysis, only 2 variables were identified as independent factors associated with infection by FRCP: diabetes mellitus $(p=0.013)$ and parenteral nutrition $(p=0.059)$. After multivariate analysis, only diabetes remained an independent factor associated with infection by FRCP $(p=0.001$ OR 1.3-3.5).

The overall candidemia mortality rates at 30 days after diagnosis were $45 \%$ (18/40) for all patients, $47.4 \%$ (9/ $19)$ for the group infected by FSC and $42.9 \%$ (9/21) for the group infected by FRCP $(p=0.8)$. Of note, liposomal amphotericin $B$ was the major therapeutic option employed for the treatment of patients infected by FRCP (12 out 21 patients, $57 \%$ ).

\section{Discussion}

This study is the first to describe an outbreak of candidemia caused by fluconazole-resistant C. parapsilosis strains in ICU patients of a tertiary care hospital in Brazil. It is important to note that the $C$. parapsilosis strains were identified by molecular methods and the phenotype of resistance to fluconazole was examined by 2 different methods, including the CLSI BMD assay.

The outbreak of $C$. parapsilosis was confirmed by a sharp increase in the incidence rate of candidemia reported in the ICU as well as by molecular typing of the isolates by microsatellite technique (please, see data about molecular typing on reference Souza et al. 2015).

During the study period, hospital physicians worked under the reasonable assumption that most episodes of C. parapsilosis candidemia were completely refractory to treatment with fluconazole (personal communication). As a consequence, the hospital staff decided to use liposomal amphotericin $\mathrm{B}$ (L-AMB) as the antifungal drug of choice to treat all episodes of $C$. parapsilosis candidemia. This decision was based on the putative limitations of echinocandins against C. parapsilosis infections and 
Table 1 Epidemiological and clinical characteristics of 40 ICU patients with fungemia caused by fluconazole-resistant C. parapsilosis (FRCP) and fluconazole-susceptible Candida species (FSC)

\begin{tabular}{|c|c|c|c|}
\hline & $\operatorname{FRCP}(N=21)$ & $\operatorname{FSC}^{\mathrm{a}}(N=19)$ & $P$ value \\
\hline Age (years) - median (range) & $70(23-91)$ & $76(35-90)$ & 0.311 \\
\hline Gender female - n (\%) & $7(33.3)$ & $8(42.1)$ & 0.567 \\
\hline Time to candidemia - median/range & $22(0-83)$ & $25(7-134)$ & 0.185 \\
\hline Apache II score - median/range & $14(3-29)$ & $16(3-30)$ & 0.138 \\
\hline Death up to 30 days after candidemia (\%) & $9(42.9)$ & $9(47.4)$ & 0.78 \\
\hline \multicolumn{4}{|l|}{ Comorbidities - n (\%) } \\
\hline Cancer & $5(23.8)$ & $2(10.5)$ & 0.270 \\
\hline Pulmonary disease & $9(42.9)$ & $11(57.9)$ & 0.342 \\
\hline Cardiac disease & $6(28.6)$ & $3(15.8)$ & 0.557 \\
\hline Diabetes mellitus ${ }^{\mathrm{b}}$ & $11(52.4)$ & $2(10.5)$ & 0.013 \\
\hline Renal failure & $4(19.0)$ & $3(15.8)$ & 1.000 \\
\hline \multicolumn{4}{|c|}{ Previous exposure to medical procedures and medicines - $\mathrm{n}(\%)$} \\
\hline Short-term catheter & $21(100.0)$ & $17(89.5)$ & 0.424 \\
\hline Catheter tip positive & $4(19.0)$ & $3(15.8)$ & 1.000 \\
\hline Previous hemodialysis & $3(14.3)$ & $1(5.3)$ & 0.673 \\
\hline Prior surgery & $12(61.9)$ & $10(52.6)$ & 0.775 \\
\hline Mechanical ventilation & $15(71.4)$ & $14(73.7)$ & 0.873 \\
\hline Parenteral nutrition & $7(33.3)$ & $1(5.3)$ & 0.059 \\
\hline Previous Hospitalization & $4(19)$ & $8(42.1)$ & 0.112 \\
\hline Antibiotics & $10(47.6)$ & $8(42.1)$ & 0.726 \\
\hline Steroids & $8(38.1)$ & $7(36.8)$ & 0.942 \\
\hline Any antifungal drug & $7(33.3)$ & $3(15.8)$ & 0.361 \\
\hline Fluconazole & $4(19.0)$ & $3(15.8)$ & 1.000 \\
\hline
\end{tabular}

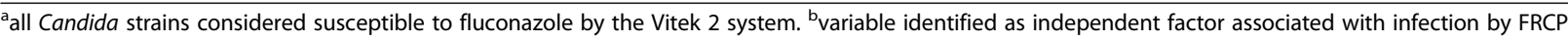

the good activity of L-AMB against biofilm-producing strains of Candida species [14, 24].

Despite treatment with L-AMB, the crude mortality rate of candidemia due to fluconazole resistant C. parapsilosis was $42.9 \%$. Indeed, this finding contradicts the current consensus that the mortality rate due to infection with C. parapsilosis is usually lower than those due to infection with C. albicans or C. tropicalis [13]. The high mortality rates observed with our casuistic study of FRCP candidemia is probably secondary to the high percentage of elderly patients $(70 \%>60$ years) as well as the high Apache II score related to those patients (see Table 1).

Although uncommon, invasive infections due to FRCP isolates have been increasingly reported. In accordance with recent studies, using antifungal susceptibility tests that are considered gold standards (CLSI and EUCASTEuropean committee on Antimicrobial Susceptibility Testing, microbroth assays), the rates of fluconazole resistance in blood stream $C$. parapsilosis isolates ranges from 3.4 to $7.5 \%$ in the USA [17, 25, 26], 0 to $6.3 \%$ in Europe and up to 5.4 in Asia $[17,27,28]$.
In Latin America, C. parapsilosis blood stream isolates have rarely been reported to be resistant to fluconazole. A recent study evaluating a total of 672 episodes of candidemia in 21 medical centers from 7 countries in Latin America found that the fluconazole MIC value for $90 \%$ of 178 strains of $C$ parapsilosis was $1.0 \mathrm{mg} / \mathrm{mL}$, and only 2 strains exhibited susceptibility-dose dependence against this drug [5]. In Brazil, the largest multicenter study ever published on the epidemiology of candidemia evaluated a total of 712 episodes of candidemia and found that all 146 C. parapsilosis strains tested were susceptible to azoles [7]. Based on those studies we can state with certainty that the present series of FRCP candidemia represents the largest experience with this emergent pathogen in our region.

C. parapsilosis (sensu stricto) has been described as the most prevalent $C$. parapsilosis complex species in both superficial and invasive human infections, and $C$. orthopsilosis and C. metapsilosis are responsible for less than $10 \%$ of the C. parapsilosis group infections in reported in different series [29-35]. Accordingly, 13 out of 14 of our C. parapsilosis isolates available 
for molecular identification were identified as $C$. parapsilosis (sensu stricto).

It is well established that C. parapsilosis is a commensal of human skin that can be transmitted horizontally via contaminated external sources and hands of health care workers [36]. We tried to identify risk factors associated with episodes of candidemia caused by FRCP, but our findings were limited by the small population size and casuistic nature of this study as well as the absence of environmental sampling data and the absence of a survey of Candida colonization among patients and health care workers. By multivariate analysis, diabetes was the only medical condition found to be independently associated with FRCP. In the present study, central catheters were not associated with FRCP; furthermore, they were almost universally present in the patients who developed candidemia, regardless of whether the causative Candida strain was susceptibility to fluconazole (97.5\%, 39 of 40 episodes). However, considering that most patients with FRCP fungemia were not previously exposed to fluconazole, we still propose that the present outbreak was probably a series of catheter-related fungemias that occurred when a resistant strain of $C$ parapsilosis was exogenously acquired by the patients during their hospitalization (15 to 40 days before developing candidemia) through contact with health care workers that were putatively colonized by this emergent pathogen.

The identification of diabetes as a risk factor of infection by FRCP in our series may be explained by two aspects: (i) this medical condition may increase the number of opportunities and the intensity of physical contact between health care workers and patients to appropriately monitor this metabolic disease both clinically and through laboratory tests; (ii) diabetic patients are more prone to colonization by $S$. aureus and eventually Candida, facilitating a putative episode of colonization by a hospital acquired fluconazole-resistant Candida strain.

\section{Conclusion}

We were able to report the first outbreak of fluconazole resistant $C$ parapsilosis strains involving adult ICU patients without a previous history of long exposition to triazoles. The clustering of incident cases in the ICU and molecular typing of strains strongly suggested the horizontal transmission of FRCP. Despite apparently uncommon, resistance of $C$ parapsilosis to fluconazole has been increasingly reported and accurate vigilant monitoring for new nosocomial strains of FRCP is required.

\section{Abbreviations}

BMD, broth microdilution; CLSI, Clinical and Laboratory Standards Institute; EUCAST, European committee on Antimicrobial Susceptibility Testing; FRCP, fluconazole-resistant C. parapsilosis; FSC, fluconazole-susceptible Candida; ICU, intensive care unit; L-AMB, Liposomal amphotericin B

\section{Acknowledgements}

We Thank Fabiola Fernandes dos Santos Castro (microbiology laboratory of Santa Luzia' Hospital) for storing the strains of Candida parapsilosis.

\section{Funding}

This study was supported by the Fundação de Amparo a Pesquisa do Estado de São Paulo (FAPESP, Grant 2012/04767-1) and Conselho Nacional de Desenvolvimento Científico e Tecnológico , Brazil, (CNPq, Grant 307510/ 2015-8). A.C.R.S. received a doctoral fellowship from FAPESP (2012/04769-4). A.L.C. received grants from FAPESP and CNPq.

Availability of data and materials

All data supporting these findings is contained within the manuscript.

\section{Authors' contributions}

Conceived and designed study: HMSP, ALC, LAC. Collection of data: HMSP, RAS, CMGD, ACRS. Analyzed the data: ALC, HMSP, LAC, RAS. Manuscript preparation: ALC, HMSP, LAC ACRS, CMGD. All authors read and approved the final manuscript.

\section{Authors' information}

HMSP: Associate Professor of Escola Superior de Ciências da Saúde, Hospital de Base do Distrito Federal, Brasília DF, Brazil.

LAC: University Hospital of Universidade de Brasília, Brasília-DF, Brazil. RAS: University Hospital of Universidade de Brasília, Brasília-DF, Brazil. ACRS: Graduate student, LEMI - Laboratório Especial de Micologia. CGD: Hospital Infection Control Service of Hospital Santa Luzia, SHLS 716, conjunto E, Zip code 70390-903 Brasilia DF, Brazil.

ALC: Professor of Medicine, Head of LEMI - Laboratório Especial de Micologia.

\section{Competing interests}

ALC has received educational grants from Astellas, Gilead, Pfizer and United Medical and research grants from Pfizer. HMSP has received educational grants from Norvartis. The other authors declare that they have no competing interests.

\section{Consent for publication}

All authors have read and agreed to Biomed requirements on publications ethics, and conduct, reporting, editing, and publications recommendations. The manuscript conforms to the journal's policies.

\section{Ethics approval and consent to participate}

The study was approved by the research ethics board of Hospital Santa Luzia as protocol number (CEP: 73/2011). This study was exempted from the request of the patient consent.

\section{Author details}

${ }^{1}$ Ed. América Office Tower, SCN Q 1 BL F, - sala-1016, Asa Norte, Brasília, DF 70711-905, Brazil. Universidade Federal de São Paulo, Rua Pedro de Toledo, 669, quinto andar, São Paulo, SP 04039-032, Brazil.

Received: 20 December 2015 Accepted: 8 August 2016

Published online: 20 August 2016

References

1. Falagas ME, Roussos N, Vardakas KZ. Relative frequency of albicans and the various non-albicans Candida spp among candidemia isolates from inpatients in various parts of the world: a systematic review. Int J Infect Dis. 2010;14:e954-66.

2. Pfaller MA, Messer SA, Moet GJ, Jones RN, Castanheira M. Candida bloodstream infections: comparison of species distribution and resistance to echinocandin and azole antifungal agents in Intensive Care Unit (ICU) and non-ICU settings in the SENTRY Antimicrobial Surveillance Program (2008-2009). Int J Antimicrob Agents. 2011:38:65-9.

3. Yapar N. Epidemiology and risk factors for invasive candidiasis. Ther Clin Risk Manag. 2014;10:95-105

4. Pfaller MA, Diekema DJ. Epidemiology of invasive candidiasis: a persistent public health problem. Clin Microbiol Rev. 2007;20:133-63.

5. Nucci M, Queiroz-Telles F, Alvarado-Matute T, Tiraboschi IN, Cortes J, Zurita $J$, et al. Epidemiology of candidemia in Latin America: a laboratory-based survey. PLoS One. 2013:8:e59373. 
6. Moretti ML, Trabasso P, Lyra L, Fagnani R, Resende MR, de Oliveira Cardoso LG, et al. Is the incidence of candidemia caused by Candida glabrata increasing in Brazil? Five-year surveillance of Candida bloodstream infection in a university reference hospital in southeast Brazil. Med Mycol. 2013;51:225-30.

7. Colombo AL, Nucci M, Park BJ, Nouér SA, Arthington-Skaggs B, da Matta DA, et al. Epidemiology of Candidemia in Brazil: a nationwide sentinel surveillance of candidemia in eleven medical centers. J Clin Microbiol. 2006; 44:2816-23.

8. Heimann SM, Cornely OA, Wisplinghoff H, Kochanek M, Stippel D, Padosch SA, et al. Candidemia in the intensive care unit: analysis of direct treatment costs and clinical outcome in patients treated with echinocandins or fluconazole. Eur J Clin Microbiol Infect Dis. 2014;34:331-8.

9. Falagas ME, Apostolou KE, Pappas VD. Attributable mortality of candidemia: a systematic review of matched cohort and case-control studies. Eur J Clin Microbiol Infect Dis. 2006;25:419-25

10. Pfaller MA, Diekema DJ, Gibbs DL, Newell VA, Ellis D, Tullio V, et al. Results from the Artemis DISK Global Antifungal Surveillance Study, 1997 to 2007: a 10.5-year analysis of susceptibilities of Candida species to Fluconazole and Voriconazole as determined by CLSI standardized disk diffusion. J Clin Microbiol. 2010:48:1366-77.

11. Hinrichsen SL, Falcão É, Vilella TAS, Colombo AL, Nucci M, Moura L, et al. Candidemia in a tertiary hospital in northeastern Brazil. Rev Soc Bras Med Trop Agosto De. 2008;41:394-8.

12. Morii D, Seki M, Binongo JN, Ban R, Kobayashi A, Sata M, Hashimoto S, Shimizu J, Morita S, Tomono K. Distribution of Candida species isolated from blood cultures in hospitals in Osaka. Japan J Infect Chemother. 2014;20:558-62.

13. Pfaller MA, Andes DR, Diekema DJ, Horn DL, Reboli AC, Rotstein C, et al. Epidemiology and outcomes of invasive candidiasis due to non-albicans species of Candida in 2,496 patients: data from the Prospective Antifungal Therapy (PATH) Registry 2004-2008. PLoS One. 2014;9:e101510.

14. Cornely OA, Bassetti M, Calandra T, Garbino J, Kullberg BJ, Lortholary O, et al. ESCMID* guideline for the diagnosis and management of Candida diseases 2012: non-neutropenic adult patients. ClinMicrobiol Infect. 2012;18:19-37.

15. Colombo AL, Guimarães T, Camargo LF, Richtmann R, de Queiroz-Telles F, Salles MJ, et al. Brazilian guidelines for the management of candidiasis - a joint meeting report of three medical societies: Sociedade Brasileira de Infectologia, Sociedade Paulista de Infectologia and Sociedade Brasileira de Medicina Tropical. Braz J Infect Dis. 2013;17:283-312.

16. Pappas PG, Kauffman CA, Andes D, Benjamin DK, Calandra TF, Edwards JE, et al. Clinical practice guidelines for the management of candidiasis: 2009 update by the Infectious Diseases Society of America. Clin Infect Dis. 2009; 48:503-35.

17. Pfaller MA, Messer SA, Woosley LN, Jones RN, Castanheira M. Echinocandin and triazole antifungal susceptibility profiles for clinical opportunistic yeast and Mold isolates collected from 2010 to 2011: application of New CLSI clinical breakpoints and epidemiological Cutoff values for characterization of geographic and temporal trends of antifungal resistance. J ClinMicrobiol. 2013;51:2571-81.

18. Souza ACR, Fuchs BB, Pinhati HMS, Siqueira RA, Hagen F, Meis JF, et al Candida parapsilosis Resistance to Fluconazole: Molecular Mechanisms and In Vivo Impact in Infected Galleria mellonella Larvae. Antimicrob Agents Chemother. 2015;59(10):6581-7.

19. Jain $P$, Khan ZK, Bhattacharya E, Ranade $S A$. Variation in random amplified polymorphic DNA (RAPD) profiles specific to fluconazole-resistant and -sensitive strains of Candida albicans. Diagn Microbiol Infect Dis. 2001;41:113-9.

20. Souza ACR, Ferreira RC, Gonçalves SS, Quindós G, Eraso E, Bizerra FC, et al. Accurate identification of Candida parapsilosis (sensu lato) by use of mitochondrial DNA and real-Time PCR. J Clin Microbiol. 2012;50:2310-4.

21. Clinical and Laboratory Standards Institute. Reference method for broth dilution antifungal susceptibility testing of yeasts; approved standard-3rd ed. document M27-A3. Wayne: CLSI; 2008

22. Clinical and Laboratory Standards Institute. Reference method for broth dilution antifungal susceptibility testing of yeasts. 4th informational supplement.document M27-S4. Wayne: CLSI; 2012

23. Barros AJ, Hirakata VN. Alternatives for logistic regression in cross-sectional studies: an empirical comparison of models that directly estimate the prevalence ratio. BMC Med Res Methodol. 2003;3:21.

24. Tumbarello M, Posteraro B, Trecarichi EM, Fiori B, Rossi M, Porta R, et al. Biofilm production by Candida species and inadequate antifungal therapy as predictors of mortality for patients with candidemia. J Clin Microbiol. 2007:45:1843-50.
25. Cleveland AA, Farley MM, Harrison LH, Stein B, Hollick R, Lockhart SR, et al. Changes in incidence and antifungal drug resistance in candidemia: results from population-based laboratory surveillance in Atlanta and Baltimore, 2008-2011. Clin Infect Dis. 2012;55:1352-61.

26. Pfaller MA, Jones RN, Castanheira M. Regional data analysis of Candida nonalbicans strains collected in United States medical sites over a 6-year period, 2006-2011. Mycoses. 2014;57:602-11.

27. Arendrup MC, Dzajic E, Jensen RH, Johansen HK, Kjældgaard P, Knudsen JD, et al. Epidemiological changes with potential implication for antifungal prescription recommendations for fungaemia: data from a Nationwide fungaemia surveillance programme. Clin Microbiol Infect. 2013;19:E343-53.

28. Minea B, Nastasa V, Moraru RF, Kolecka A, Flonta MM, Marincu I, et al. Species distribution and susceptibility profile to fluconazole, voriconazole and MXP-4509 of 551 clinical yeast isolates from a Romanian multi-centre study. Eur J Clin Microbiol Infect Dis. 2015;34:367-83.

29. Silva AP, Miranda IM, Lisboa C, Pina-Vaz C, Rodrigues AG. Prevalence, distribution, and antifungal susceptibility profiles of Candida parapsilosis, C. orthopsilosis, and C. Metapsilosis in a tertiary care hospital. J Clin Microbiol. 2009;47:2392-7.

30. Gonçalves SS, Amorim CS, Nucci M, Padovan ACB, Briones MRS, Melo ASA, et al. Prevalence rates and antifungal susceptibility profiles of the Candida parapsilosis species complex: results from a nationwide surveillance of candidaemia in Brazil. Clin Microbiol Infect. 2010;16:885-7.

31. Ruiz LS, Khouri S, Hahn RC, da Silva EG, de Oliveira VK, Gandra RF, et al. Candidemia by species of the Candida parapsilosis complex in children's hospital: prevalence, biofilm production and antifungal susceptibility. Mycopathologia. 2013;175:231-9.

32. Bonfietti LX, Martins Mdos A, Szeszs MW, Pukiskas SB, Purisco SU, Pimentel FC, et al. Prevalence, distribution and antifungal susceptibility profiles of Candida parapsilosis, Candida orthopsilosis and Candida metapsilosis bloodstream isolates. J Med Microbiol. 2012;61:1003-8.

33. Trabasso P, Matsuzawa T, Fagnani R, Muraosa Y, Tominaga K, Resende MR, et al. Isolation and drug susceptibility of Candida parapsilosis sensu lato and other species of C. parapsilosis complex from patients with blood stream infections and proposal of a novel LAMP identification method for the species. Mycopathologia. 2015;179:53-62.

34. Cantón E, Pemán J, Quindós G, Eraso E, Miranda-Zapico I, Álvarez M, et al. Prospective multicenter study of the epidemiology, molecular identification, and antifungal susceptibility of Candida parapsilosis, Candida orthopsilosis, and Candida metapsilosis isolated from patients with candidemia. Antimicrob Agents Chemother. 2011;55:5590-6.

35. Feng $X$, Ling B, Yang G, Yu X, Ren D, Yao Z. Prevalence and distribution profiles of Candida parapsilosis, Candida orthopsilosis and Candida metapsilosis responsible for superficial candidiasis in a Chinese university hospital. Mycopathologia. 2012;173:229-34.

36. Trofa D, Gácser A, Nosanchuk JD. Candida parapsilosis, an emerging fungal pathogen. Clin Microbiol Rev. 2008;21:606-25.

\section{Submit your next manuscript to BioMed Central and we will help you at every step:}

- We accept pre-submission inquiries

- Our selector tool helps you to find the most relevant journal

- We provide round the clock customer support

- Convenient online submission

- Thorough peer review

- Inclusion in PubMed and all major indexing services

- Maximum visibility for your research

Submit your manuscript at www.biomedcentral.com/submit 